\title{
On boundary conditions for spin-diffusion equations with Rashba spin-orbit interaction
}

\author{
O.Bleibaum* \\ Institute for Theoretical Physics, Otto-von-Guericke University, \\ 39016 Magdeburg, PF4120, Germany
}

\begin{abstract}
We reexamine the boundary conditions of spin diffusion equations for dirty semiconductor heterostructures with weak linear Rashba spin-orbit interaction. Doing so, we focus on the influence of tangent derivatives of the particle density at the boundary on the magnetization. Such derivatives are associated with a spin accumulation in the presence of a density gradient. We show that the tangent derivatives enter the boundary conditions and argue that the spin-Hall effect is absent in such systems because of this fact.
\end{abstract}




\section{INTRODUCTION}

At present there is much interest in spin-charge coupling effects in non-magnetic semiconductor heterostructures with linear Rashba spin-orbit interaction, like the spin accumulation in an external electric field (see, e.g., Refs. 1, 2, 3, 4]), spin-galvanic currents ${ }^{5.6}$ or the spin-Hall effect (see, e.g., 7, 8, 9, 10]). While the first two effects can also be observed in homogeneous systems the spin-Hall effect manifests itself in a non-equilibrium magnetization at the boundaries of a sample in the presence of electric fields. Thus, investigations of the magnetization at the sample boundary require the development of a transport theory, which is also able to describe the evolution of particle and spin packets.

Problems, which are related to the motion of particle packets in semiconductors, have been investigated much in the literature (see, e.g., Ref.[11]). Drift-diffusion equations are typically used to this end. A boundary enters the diffusion equations via boundary conditions, which have to be used in seeking solutions to the differential equations. These boundary conditions follow often from conservation laws, as it is the case in investigations of charge transport processes. There the boundary conditions reduce to the requirement the electric current is continuous at the boundary. This condition implies the current vanishes at hard-wall boundaries. The structure of the current follows from the conservation of the charge.

Diffusion equations for the investigation of inhomogeneous magnetizations in semiconductor heterostructures with Rashba spin-orbit interaction have been derived in a number of papers (see, e.g., Refs. 12,13,14,15,16]). However, in most cases they have been applied to systems without boundaries. The problem how to formulate boundary conditions for systems with hard-wall boundary is a rather controversial one. The conventional conservation laws can not be invoked, since the magnetization is not a conserved quantity in the systems in question. To circumvent this problem some researchers have taken the point view that the spin current can be extracted from the derivative terms in the diffusion equation ${ }^{13.16}$. The latter takes the form 16

$$
\begin{aligned}
\partial_{t} \boldsymbol{S} & +\boldsymbol{\Omega} \circ\left(\boldsymbol{S}-\boldsymbol{S}_{0}\right)+\boldsymbol{R} \times\left(\boldsymbol{S}-\boldsymbol{S}_{0}\right)-D \Delta \boldsymbol{S} \\
& +D(\boldsymbol{F} \cdot \boldsymbol{\nabla}) \partial_{\epsilon} \boldsymbol{S}-\omega_{s}(\boldsymbol{N} \times \boldsymbol{\nabla}) \times\left(\boldsymbol{S}-\boldsymbol{S}_{0}\right)=-\partial_{\epsilon} \boldsymbol{j}_{\epsilon}
\end{aligned}
$$

in the presence of a constant electric field $\boldsymbol{F}$ ( $\boldsymbol{S}$ is the spin density, $D$ is the diffusion constant, $\boldsymbol{\Omega}$ is a symmetric tensor of second rank, $\circ$ is the dyadic product, $\boldsymbol{R}=\boldsymbol{F} \times \boldsymbol{N} \partial_{\epsilon} \omega_{s} / 2$ is a vector, $\omega_{s}=4 \mathrm{mD} / \hbar$ is a transport coefficient, $\boldsymbol{N}=N \boldsymbol{e}_{z}$ is a vector perpendicular to the plane of the two-dimensional electron gas, $\partial_{\epsilon}$ is the derivative with respect to energy, $\boldsymbol{j}_{\epsilon}$ is the energy current for spins and $\boldsymbol{S}_{0}=-\tau \boldsymbol{N} \times \boldsymbol{\nabla} n+\tau \boldsymbol{N} \times \boldsymbol{F} \partial_{\epsilon} n$ is the spin accumulation, which can be induced either by the constant field $\boldsymbol{F}$ or by a gradient of the density $n$ ). A characteristic feature of this equation is the fact most of its terms depend only on the deviation of the magnetization from the spin accumulation $\boldsymbol{S}_{0}$. Thus, the point view that all derivative terms are part of the divergence of the spin-current tensor leads to the conclusion that the derivative of the magnetization normal to the boundary is proportional to $\mathbf{S}-\mathbf{S}_{0}$ and thus zero in the stationary state. Consequently, there is no spin-Hall effect in such systems in the presence of a hard-wall boundary. This point of view is supported further by the fact that no anomalous Hall-current is induced by injection of particles with perpendicular spin in electric fields 16 .

However, although diffusion currents have been extracted from diffusion equations for centuries it has to be said that this approach is problematic. The diffusion equations contain only the divergence of the spin-current tensor, so terms proportional to a curl can not be 
retrieved unambiguously by this procedure. Moreover, the interpretation of terms as part of the divergence of a tensor of second rank is also ambiguous. The sixth term on the left hand side (lhs) of Eq.(11), e.g., can also be decomposed into the gradient of a scalar field and the curl of a vector field. This raises the question, whether there is another procedure, which can be used to determine boundary conditions for diffusion equations.

Recently, such a method has been developed in Ref.[17]. Starting from the notion, that the boundary conditions for the system in question can be expressed in the form

$$
\boldsymbol{e}_{n} \cdot \nabla \rho_{\alpha}=B_{\alpha \beta} \rho_{\beta}
$$

which connects the normal derivative $\boldsymbol{e}_{n} \cdot \boldsymbol{\nabla} \rho_{\alpha}$ of the densities $\rho_{\alpha}=(n, \boldsymbol{S})$ with the densities themselves, they calculate the coefficients $B_{\alpha \beta}$ from the integral equations for the densities (A summation with respect to double indices must be performed in all equations.). Doing so, they use the fact that the integrals are determined by two scales near the boundary, by the Fermi wave-length and the mean free path. The first length is the distance from the boundary needed to approach a constant particle density and the second the distance needed to feel the impact of disorder. Thus, transport is quasi-ballistic in the intermediate region. After a tour the force through rather complicated integrals they find that the coefficients coupling spin and charge vanish in the intermediate region and that those coupling spin and spin are non-zero. This fact has a very important consequence. The derivatives of the magnetization do not vanish at the boundary in this case, since the magnetization tends to approach the value $\boldsymbol{S}_{0}$ in the stationary limit (see Eq.(11)). Therefore, spin-Hall effect is obtained.

The method developed in Ref. [17] has proven to be very useful in the investigation of the boundary conditions. The results of Ref. 17], however, are neither in line with those of previous investigations of the structure of the diffusion equations ${ }^{16}$ nor with the investigations of the spin-current tensor. Already a glance on the spin-diffusion equations (11) reveals we should expect that the boundary conditions are not determined by the magnitude of the magnetization but by the deviation of the magnetization from the spin accumulation $\mathbf{S}_{0}$. The spin accumulation, however, is determined by tangent derivatives. Such derivatives are not contained in Eq.(2). Thus, the ansatz (2) is simply not sufficiently general enough to accommodate the spin accumulation. To allow for an impact of the spin accumulation we should replace the ansatz (2) by the new ansatz

$$
\boldsymbol{e}_{n} \cdot \nabla \rho_{\alpha}=B_{\alpha \beta} \rho_{\beta}+A_{\alpha \beta} \boldsymbol{e}_{t} \cdot \nabla \rho_{\beta}
$$

and determine the spin-charge coupling coefficients of the matrix $A_{\alpha \beta}$ ( $\boldsymbol{e}_{t}$ is the unit tangent vector). Boundary conditions of the type (3) are not unusual for partial differential equations. Indeed, also the boundary conditions for Maxwells equations can be formulated in terms of normal and tangent derivatives of fields.

Motivated by our observation we now calculate the spin-charge coupling coefficients of the matrix $A_{\alpha \beta}$ for systems with weak Rashba interaction in the absence of external fields. To this end we focus on the situation in a half space and assume that there is a gradient in the charge carrier density tangent to the boundary. Moreover, we assume that the normal derivative of the charge carrier density vanishes. This is just the situation in a spin-Hall experiment. The spin-spin coupling coefficients are of higher order with respect to the Rashba interaction and therefore negligible in the systems in question. 


\section{BASIC EQUATIONS}

The Hamilton operator for the two-dimensional electron gas has the form

$$
H=\frac{\hat{\boldsymbol{p}}^{2}}{2 m}+\boldsymbol{N} \cdot(\boldsymbol{\sigma} \times \hat{\boldsymbol{p}})+V(\boldsymbol{x}) .
$$

Here $\hat{\boldsymbol{p}}$ is the momentum operator, $\boldsymbol{\sigma}$ is the spin-operator and $V(\boldsymbol{x})$ is a random potential with zero average, Gaussian statistics and mean squared deviation

$$
\left\langle V(\boldsymbol{x}) V\left(\boldsymbol{x}^{\prime}\right)\right\rangle=\frac{\hbar}{2 \pi \nu \tau} \delta\left(\boldsymbol{x}-\boldsymbol{x}^{\prime}\right)
$$

The unit vectors in the 2-d plane are $\boldsymbol{e}_{x}$ and $\boldsymbol{e}_{y}$. $\boldsymbol{e}_{z}$ is a unit vector perpendicular to the plane. To be specific we consider the half-space $y>0$. Thus, derivatives with respect to $y$ are normal to the boundary and derivatives with respect to $x$ are tangent to the boundary. The labels $x, y$ and $z$ are replaced by the indices $1,2,3$ in sums. Latin indices run from 1-3, Greek indices from 0-3. The index zero is associated with the particle density and $\sigma_{0}$ is the unit matrix. Double indices have to be summed over in all equations.

The propagation of single particle excitations is described by the retarded and advanced Green's functions. To calculate them we use the fact that the system is translation invariant in $x$-direction in average, so we we can use the Fourier transformation. Doing so, we find that the retarded $(\mathrm{R})$ Green's functions is given by

$$
G_{0}^{R}\left(y, y^{\prime} \mid k\right)=\frac{m}{\lambda_{R} \hbar^{2}}\left(e^{-\lambda_{R}\left(y+y^{\prime}\right)}-e^{-\lambda_{R}\left|y-y^{\prime}\right|}\right)
$$

in the absence of the spin-orbit interaction, where

$$
\lambda_{R}=-i \sqrt{k_{F}^{2}-k^{2}+\frac{i k_{F}}{l}} .
$$

Here $\hbar k_{F}$ is the momentum at the Fermi surface and $l$ is the mean free path. The advanced (A) Green's function is obtained from Eq.(6) by hermitian conjugation. To take into account the spin-orbit interaction we use the Dyson equation approach of Ref.[17]. The spin-orbit interaction is considered as the perturbation in this approach and Eq.(6) as the leading approximation. Thus, the approach ignores the impact of the boundary on the scattering time. The first correction with respect to the spin-orbit interaction is given by $G_{1}^{R}=$ $\delta_{1} G_{1}^{R}+\delta_{2} G_{1}^{R}$, where

$$
\delta_{1} G_{1}^{R}\left(y, y^{\prime} \mid k\right)=-\sigma_{y} \frac{N k m^{2}}{\lambda_{R}^{2} \hbar^{3}}\left[e^{-\lambda_{R}\left|y-y^{\prime}\right|}\left(\left|y-y^{\prime}\right|+\frac{1}{\lambda_{R}}\right)-e^{-\lambda_{R}\left(y+y^{\prime}\right)}\left(y+y^{\prime}+\frac{1}{\lambda_{R}}\right)\right]
$$

and

$$
\delta_{2} G_{1}^{R}\left(y, y^{\prime} \mid k\right)=\sigma_{x} \frac{i m^{2} N}{\lambda_{R} \hbar^{3}}\left(y-y^{\prime}\right)\left[e^{-\lambda_{R}\left|y-y^{\prime}\right|}-e^{-\lambda_{R}\left(y+y^{\prime}\right)}\right] .
$$

This correction is sufficient to calculate the matrix $B_{\alpha \beta}$ for systems with weak Rashba interaction. Therefore, the consideration has been restricted to this contribution in previous calculations $^{17}$. The investigation of spin-charge coupling effects, however, requires to go beyond this approximation. The leading contributions to these coefficients result from corrections of second order with respect to the spin-orbit interaction odd in $k$ in the situation 
discussed here. Therefore, we also need the odd part of the second order correction to the Green's function. It is given by

$$
\left.G_{2}^{R}\left(y, y^{\prime} \mid k\right)\right|_{\text {odd }}=\frac{2 N^{2} k m^{3}}{\lambda_{R}^{2} \hbar^{4}} y y^{\prime} \sigma_{z} e^{-\lambda_{R}\left(y+y^{\prime}\right)} .
$$

\section{THE BOUNDARY CONDITIONS}

To extract the boundary conditions we use the fact that the densities satisfy the system of integral equations

$$
\rho_{\alpha}(\boldsymbol{x})=\frac{\hbar}{4 \pi \nu \tau} \int d \boldsymbol{x}_{1} \operatorname{tr}\left(\sigma_{\alpha} G^{R}\left(\boldsymbol{x}, \boldsymbol{x}_{1}\right) \sigma_{\beta} G^{A}\left(\boldsymbol{x}_{1}, \boldsymbol{x}\right)\right) \rho_{\beta}\left(\boldsymbol{x}_{1}\right)
$$

in the stationary limit. Here the Green's functions are those in position representation. To simplify this expression we expand the integrals at the point $\boldsymbol{x}$ and obtain

$$
\begin{aligned}
\rho_{\alpha}(\boldsymbol{x})= & \rho_{\beta}(\boldsymbol{x}) \frac{\hbar}{4 \pi \nu \tau} \int_{0}^{\infty} d y_{1} \int \frac{d k}{2 \pi} \operatorname{tr}\left(\sigma_{\alpha} G^{R}\left(y, y_{1} \mid k\right) \sigma_{\beta} G^{A}\left(y_{1}, y \mid k\right)\right) \\
& +\partial_{y} \rho_{\beta}(\boldsymbol{x}) \frac{\hbar}{4 \pi \nu \tau} \int_{0}^{\infty} d y_{1} \int \frac{d k}{2 \pi}\left(y_{1}-y\right) \operatorname{tr}\left(\sigma_{\alpha} G^{R}\left(y, y_{1} \mid k\right) \sigma_{\beta} G^{A}\left(y_{1}, y \mid k\right)\right) \\
& +\partial_{x} \rho_{\beta}(\boldsymbol{x}) \frac{\hbar}{4 \pi \nu \tau} \int_{0}^{\infty} d y_{1} \int \frac{d k}{2 \pi} \operatorname{tr}\left(\sigma_{\alpha} G^{R}\left(y, y_{1} \mid k\right) \sigma_{\beta} i \partial_{k} G^{A}\left(y_{1}, y \mid k\right)\right) .
\end{aligned}
$$

The first two terms on the right hand side (rhs) of Eq.(12) are those considered in Ref. 17]. The third term is the new term associated with the tangent derivative of the fields. To calculate the integrals we choose a point $\boldsymbol{x}$, which satisfies the conditions $k_{F} y \gg 1$ and $y / l \ll 1$. Doing so, we find that Eq.(12) can be written in the form

$$
\begin{gathered}
0=-D \partial_{y} S_{x}, \quad 0=-D \partial_{y} S_{y}-\frac{1}{2} N \omega_{s} S_{z}, \\
0=-D \partial_{y} S_{z}+\frac{1}{2} N \omega_{s}\left(S_{y}-S_{0 y}\right),
\end{gathered}
$$

where $D$ is the diffusion coefficient and $S_{0 y}$ is the spin accumulation induced by the density gradient. We would like to mention that the terms proportional to the magnetization in the Eqs.(13) and (14) agree with those discussed in Ref.[17]. The second term in the bracket of Eq.(14), however, is a new term, which couples spin and charge. Eq.(14) shows, that the normal derivative of the magnetization depends only on the deviation of the magnetization from the spin accumulation $\boldsymbol{S}_{0}$. Thus, there is no spin-Hall effect in systems with hard-wall boundary, as detailed in Ref.[16].

We would like to mention that the use of the Fourier-transformed Green's functions simplifies the integrals strongly. If we use the Eqs.(6)-(10) we obtain a number of integrals, which contribute to Eq.(12). However, none of them is as complicated as those considered in Ref. 17], where Green's functions in position space were used. The integrals with respect to $y_{1}$ reduce to simple exponential integrals, which can be calculated immediately. 
The remaining integrands reduce either to functions of the type $f(k)$, to functions of the type $y^{\alpha} f(k) \exp \left(-\operatorname{Re} \lambda_{R} y\right)$ or to functions of the type $y^{\alpha} \operatorname{Re}\left(f(k) \exp \left(-\lambda_{R} y\right)\right)$ (here $f(k)$ are complex functions, which do not cause problems). The latter are those, which are strongly oscillating, as discussed in Ref. 17]. All of the integrals simplify strongly if the chain of substitutions $k=k_{F} x, x^{2}-1=\epsilon \sinh (z), \exp (-z / 2)=w$ is used $\left(\epsilon=1 / k_{F} l\right)$. The intermediate scale $k_{F}^{-1} \ll y \ll l$ can immediately be recognized after these substitutions and a complete asymptotic expansion can be obtained with methods as simple as parts integration. We would like to mention, that there is also the new parameter $\sqrt{\epsilon} k_{F} y$ in the integrals. The leading contributions, however, do not depend on this parameter.

\section{ON THE STRUCTURE OF THE DIFFUSION EQUATION}

If we compare the boundary conditions (13) and (14) with those of Ref. [16] we note they differ from those of Ref. [16] by a factor $1 / 2$. Although this fact does not affect the conclusions of Ref. [16] there is the question what the source of this factor is. A glance at Eq.(11) reveals, that the factor $1 / 2$ implies, that only $1 / 2$ of the sixth term on the lhs of Eq.(11) is a part of the divergence of the spin-current tensor. Thus, we can write Eq.(11) in the form

$$
\partial_{t} \boldsymbol{S}+\boldsymbol{\Omega} \circ\left(\boldsymbol{S}-\boldsymbol{S}_{0}\right)+\tilde{\boldsymbol{R}} \times\left(\boldsymbol{S}-\boldsymbol{S}_{0}\right)+\operatorname{Div} J=-\partial_{\epsilon} \boldsymbol{j}_{\epsilon}
$$

where

$$
\operatorname{Div} J=-D \Delta \boldsymbol{S}+D(\boldsymbol{F} \cdot \boldsymbol{\nabla}) \partial_{\epsilon} \boldsymbol{S}-\frac{1}{2} \omega_{s}(\boldsymbol{N} \times \boldsymbol{\nabla}) \times\left(\boldsymbol{S}-\boldsymbol{S}_{0}\right)
$$

and

$$
\tilde{\boldsymbol{R}}=\boldsymbol{R}-\frac{1}{2} \omega_{s} \boldsymbol{N} \times \boldsymbol{\nabla}
$$

Eq.(15) differs from conventional diffusion equations for the magnetization only in that the third term on the rhs is proportional to $\boldsymbol{S}-\boldsymbol{S}_{0}$. The term $\tilde{\boldsymbol{R}} \times \boldsymbol{S}_{0}$ is absent in conventional diffusion equations, since $\tilde{\boldsymbol{R}} \| \boldsymbol{S}_{0}$ in them. Thus, the presence of this term in the diffusion equation gives rise to the notion that the impact of the field differs from that of a density gradient, as already discussed in Ref. 16]. However, if we take into account the structure of the vectors $\boldsymbol{R}$ and $\tilde{\boldsymbol{R}}$ we can also write this equation in the form

$$
\partial_{t} \boldsymbol{S}+\boldsymbol{\Omega} \circ\left(\boldsymbol{S}-\boldsymbol{S}_{0}\right)+\tilde{\boldsymbol{R}} \times \boldsymbol{S}+\operatorname{Div} J=-\partial_{\epsilon}\left(\boldsymbol{j}_{\epsilon}+\frac{1}{2} \omega_{s}(\boldsymbol{N} \times \boldsymbol{\nabla}) \times(\boldsymbol{N} \times \boldsymbol{F}) n\right) .
$$

This fact shows that the impact of the field on the system in question is the same as the impact of a density gradient, at least on the level of the diffusion equations. Thus, the fact that only $1 / 2$ of the sixth term enters the spin-current tensor restores the symmetry between the response to an electric field and the response to a density gradient. Eq.(18) also indicates that the second term on the rhs of Eq.(18) is a part of the energy relaxation current. The diffusion equations for systems with Rashba spin-orbit interaction reduce just to the conventional diffusion equations in this case.

We stress that the conjecture that the second term on the rhs of Eq.(18) is part of the energy current still needs justification. The present paper clarifies the boundary conditions in position space. The energy space is a different subject, which we hope to address in a future publication. 


\section{CONCLUSIONS}

In the present paper we have reinvestigated the boundary conditions for spin diffusion equations with Rashba spin-orbit interaction. Our investigation shows that there are also spin-charge coupling terms in the boundary conditions. They couple the tangent derivative of the particle density to the normal derivative of th spin-density. There is no spin-Hall effect in systems with weak Rashba spin-orbit interaction in the presence of a hard-wall boundary due to the spin-charge coupling terms.

\section{Acknowledgments}

The author is grateful to B. K. Nikolić for drawing his attention to Ref.[17] and to H. Böttger for many useful and stimulating discussions.

* Electronic address: olaf.bleibaum@physik.uni-magdeburg.de

1 V. M. Edelstein, Solid Sate Commun. 73, 233 (1990).

2 Y. K. Kato, R. C. Myers, A. C. Gossard, and D. D. Awschalom, Phys. Rev. Lett.93, 176601 (2004).

3 A. Yu. Silov, P. A. Blajov, J. H. Woller, R. Hey, K. H. Ploog, and N. S. Averkiev, Appl. Phys. Lett. 85, 5929 (2004).

4 J. I. Inoue, G. E. W. Bauer, and L. W. Molenkamp, Phys. Rev. B 67, 033104 (2003).

5 S. D. Ganichev, E. L. Ivchenko, S. N. Danilov, J. Eroms, W. Wegscheider, D. Weiss, W. Prettl, Phys. Rev. Lett. 86, 4358 (2001).

6 S. D. Ganichev, V. V. Belkov, L. E. Golub, E. L. Ivchenko, P. Schneider, S. Giglberger, J. Eroms, J. DeBoeck, G. Borghs, W. Wegscheider, D. Weiss, and W. Prettl, Phys. Rev. Lett. 92, 256601 (2004).

7 J. Sinova, D. Culcer, Q. Niu, N. A. Sinitsyn, T. Jungwirth, and A. H. MacDonald, Phys. Rev. Lett.92, 126603 (2004).

8 J. Wunderlich, B. Kaestner, J. Sinova, T. Jungwirth, Phys. Rev. Lett. 94, 047204 (2005).

9 B. K. Nikolić, L. P. Zabô, and S. Souma, Phys. Rev. B 72, 075361 (2005), Phys. Rev. B 73, 075303 (2006).

10 I. Adagideli and G. E. W. Bauer, Phys. Rev. Lett. 95, 256602 (2005).

11 M. Balkanski and R. F. Wallis, Semiconductor Physics and Applications, Oxford University Press (2000).

12 O. Bleibaum, Phys. Rev. B 69, 205202 (2004).

13 T. Damker, H. Böttger, V. V. Bryksin, Phys. Rev. B 69, 205327 (2004).

14 A. A. Burkov, A. S. Nunez, and A. H. MacDonald, Phys. Rev. B 70, 155308 (2004).

15 E. G. Mishchenko, A. V. Shytov, and B. I. Halperin, Phys. Rev. Lett. 93, 226602 (2004).

16 O. Bleibaum, Phys. Rev. B 73, 35322 (2006), phys. stat. sol. (b) 243, 403 (2006).

17 V. M. Galitski, A. A. Burkov and S. Das Sarma, cond-mat/0601677v2 (2006). 